\title{
Specific Features of Magnetic Structure Formation in Orbitally Degenerate $\mathrm{BiMnO}_{3}$ Manganite $^{1}$
}

\author{
L. E. Gonchar ${ }^{a, b},{ }^{*}$, T. O. Nikitina ${ }^{a}$, and A. E. Nikiforov ${ }^{a}$ \\ ${ }^{a}$ Ural Federal University, Yekaterinburg, 620002 Russia \\ ${ }^{b}$ Ural State University of Railway Transport, Yekaterinburg, 620034 Russia \\ *e-mail: lyudmila.gonchar@usu.ru
}

\begin{abstract}
The orbital structure and magnetic ordering of the Jahn-Teller multiferroic $\mathrm{BiMnO}_{3}$ manganite have been theoretically studied. It is shown that the orbital structure depends not only on the nearest-neighbor oxygen environment of manganese ions, but also on their next-to-nearest neighbors. The orbital structure significantly influences the magnetic order that forms as a result of competition between ferromagnetic and antiferromagnetic exchange interactions.
\end{abstract}

DOI: $10.1134 / \mathrm{S} 106377611305018 \mathrm{X}$

\section{INTRODUCTION}

The interest in Jahn-Teller magnetic oxides is increasing due to a number of specific effects that can be simultaneously observed in these crystals, including colossal magnetoresistance and numerous structural and magnetic phase transitions driven by temperature, magnetic and electric fields, and pressure. The unusual properties of these compounds are determined by the strong mutual relationship of crystalline, charge, orbital, and magnetic structures. In recent years, extensive research has also been devoted to multiferroelectric compounds in which magnetic order coexists with spontaneous polarization.

Bismuth manganite $\mathrm{BiMnO}_{3}$ has been treated in many investigations as a multiferroelectric compound [1-6]. However, the results of recent experiments [7, 8] have shown that this ferromagnetic crystal belongs to the $C 2 / c$ symmetry group, which excludes the existence of spontaneous polarization [5-9]. External factors (temperature, pressure) can alter the magnetic structure of $\mathrm{BiMnO}_{3}$ crystals so as to stabilize antiferromagnetism $[8,9]$. The nonstoichiometry of a crystal with respect to oxygen also strongly influences the crystalline and magnetic structures: an increase in the amount of oxygen ions leads to a change in the magnetic order from ferro- to antiferromagnetic [10].

It has been suggested that bismuth manganite, similar to many rare-earth manganites, possesses an orbital structure, competitive exchange interactions, and the related magnetic frustration [5-8]. The present work was aimed at studying the effect of orbital ordering on the magnetic structure of $\mathrm{BiMnO}_{3}$ in the presence of competitive exchange interactions.

\footnotetext{
${ }^{1}$ The article is based on a preliminary report delivered at the 36 th Conference on Low-Temperature Physics (St. Petersburg, July 2-6, 2012).
}

\section{CRYSTALLINE AND ORBITAL STRUCTURES OF $\mathrm{BiMnO}_{3}$}

According to experimental data $[7,8]$, the crystalline lattice of bismuth manganite belongs to the $C 2 / c$ symmetry group. In this monoclinic group, the vectors of the main lattice periods are not mutually orthogonal. Experimental data [8] are summarized in Tables 1 and 2.

The crystalline lattice of $\mathrm{BiMnO}_{3}$ is a distorted perovskite structure, in which $\mathrm{Mn}^{3+}$ ion in a octahedral environment has an orbitally degenerate ${ }^{5} E$ ground state. In this case, the orbital degeneracy can be removed due to the Jahn-Teller effect [10]. The existence of an orbital structure in $\mathrm{BiMnO}_{3}$ crystals was theoretically predicted [5-8]. The ground-state wavefunction on each manganese ion has the following form [11]:

$$
\psi_{n}=\sin \frac{\Phi_{n}}{2}|\theta\rangle_{n}+\cos \frac{\Phi_{n}}{2}|\varepsilon\rangle_{n}
$$

where $|\theta\rangle_{n}$ and $|\varepsilon\rangle_{n}$ are the eigenfunctions and $\Phi_{n}$ is the angle that characterizes the mixing of eigenfunctions of the ${ }^{5} E$ ground state of the $n$th ion of trivalent manganese.

The orbital structure will be described using a model of electron-vibrational (vibronic) interactions as described by the following Hamiltonian [12, 13]:

$$
H_{\text {vib }}=H_{\text {lin }}+H_{Q Q}+H_{R} \text {. }
$$

Let us consider partial contributions stipulated by this model. The main linear contribution from the nearest-neighbor oxygen environment of manganese ion can be written as

$$
H_{\mathrm{lin}}=V_{e} \sum_{n}\left(Q_{\theta n} X_{\theta n}+Q_{\varepsilon n} X_{\varepsilon n}\right)
$$


Table 1. Parameters of crystalline lattice of $\mathrm{BiMnO}_{3}$

\begin{tabular}{c|c|c|c|c|c}
\hline$a, \AA$ & $b, \AA$ & $c, \AA$ & $\alpha$ & $\beta$ & $\gamma$ \\
\hline 9.5473 & 5.6167 & 9.8699 & $90^{\circ}$ & $110^{\circ}$ & $90^{\circ}$ \\
\hline
\end{tabular}

Table 2. Coordinates (expressed in fractions of lattice parameters) of ions in $\mathrm{BiMnO}_{3}$ lattice belonging to $C 2 / c$ symmetry group

\begin{tabular}{l|l|l|l}
\hline \multicolumn{1}{c|}{ Ion } & \multicolumn{1}{c|}{$x$} & \multicolumn{1}{c}{$y$} & \multicolumn{1}{c}{$z$} \\
\hline Bi $(4 f)$ & 0.1375 & 0.2114 & 0.1269 \\
Mn1 $(2 e)$ & 0.0 & 0.219 & 0.75 \\
Mn2 $(2 d)$ & 0.25 & 0.25 & 0.5 \\
O1 $(4 f)$ & 0.097 & 0.173 & 0.578 \\
O2 $(4 f)$ & 0.145 & 0.568 & 0.366 \\
O3 $(4 f)$ & 0.356 & 0.547 & 0.166 \\
\hline
\end{tabular}

where

$$
X_{\theta n}=\left(\begin{array}{cc}
-1 & 0 \\
0 & 1
\end{array}\right), \quad X_{\varepsilon n}=\left(\begin{array}{ll}
0 & 1 \\
1 & 0
\end{array}\right)
$$

are the matrices of orbital operators constructed using the $|\theta\rangle_{n}$ and $|\varepsilon\rangle_{n}$ functions of the $n$th $\mathrm{Mn}^{3+}$ ion; $Q_{\theta n}$ and $Q_{\varepsilon n}$ are the symmetrized coordinates of $e_{g}$ type distortions of the nearest-neighbor oxygen environment of the $n$th $\mathrm{Mn}^{3+}$ ion; and $V_{e}=-1.29 \mathrm{eV} / \AA$ is the linear vibronic interaction constant determined in [14].

However, if strong distortions of the crystalline lattice of bismuth manganite are taken into account, linear contribution (3) may be insufficient to adequately describe the orbital structure. The strong influence on mixing angle $\Phi_{n}$ of the wavefunctions can be produced by interactions of some other types. According to [12, $13,15]$, these are (i) interaction with nearest neighbors in the octahedral environment of higher orders:

$$
\begin{aligned}
H_{Q Q}= & V_{b} \sum_{n}\left[\left(2 Q_{z n}^{2}-Q_{x n}^{2}-Q_{y n}^{2}\right) X_{\theta n}\right. \\
& \left.+\sqrt{3}\left(Q_{x n}^{2}-Q_{y n}^{2}\right) X_{\varepsilon n}\right] \\
+ & N_{e} \sum_{n}\left[\left(Q_{\varepsilon n}^{2}-Q_{\theta n}^{2}\right) X_{\theta n}+2 Q_{\theta n} Q_{\varepsilon n} X_{\varepsilon n}\right],
\end{aligned}
$$

where $Q_{\theta n}, Q_{\varepsilon n}$ are the symmetrized coordinates of $e_{g}$ type distortions of the nearest-neighbor oxygen environment of the $n$th $\mathrm{Mn}^{3+}$ ion and $Q_{x n}, Q_{y n}, Q_{z n}$ are the symmetrized coordinates of $t_{1 g}$ type distortions of the nearest-neighbor oxygen environment of the $n$th $\mathrm{Mn}^{3+}$ ion; and (ii) interaction with next-to-nearest neighbors ( $\mathrm{Bi}^{3+}$ ions) of the $n$th $\mathrm{Mn}^{3+}$ ion:

$$
H_{R}=V_{e}^{R} \sum_{n}\left(Q_{\theta n}^{R} X_{\theta n}+Q_{\varepsilon n}^{R} X_{\varepsilon n}\right),
$$

where $Q_{\theta n}^{R}, Q_{\varepsilon n}^{R}$ are the symmetrized coordinates of $e_{g}$ type distortions of the next-to-nearest neighbor environment $\left(\mathrm{Bi}^{3+}\right.$ ion sublattice) of the $n$th $\mathrm{Mn}^{3+}$ ion.

The constants of these additional exchange interactions $\left(V_{b}, N_{e}, V_{e}^{R}\right)$ have not been estimated, but their possible influence on the orbital and magnetic states of the crystal can be considered. Table 3 presents data for the symmetrized distortions of the environment of manganese ions as calculated from experimental data [8]. As can be seen, $t_{1 g}$ type distortions are comparable with (or even greater than) $e_{g}$ type distortions and, hence, must also be taken into account in the adopted model.

Thus, it is possible to describe the mixing of ground-state wavefunctions (1) either in an approximation of linear vibronic interaction with nearest neighbors using $\Phi_{n}$ angles (with subscript $n$ enumerating manganese ions) determined from the relations

$$
\begin{gathered}
\cos \Phi_{n}=\frac{Q_{\theta n}}{\rho_{n}}, \quad \sin \Phi_{n}=\frac{Q_{\varepsilon n}}{\rho_{n}}, \\
\rho_{n}=\sqrt{Q_{\theta n}^{2}+Q_{\varepsilon n}^{2}}
\end{gathered}
$$

or in an approximation admitting a greater number of interactions (2)-(5) using the $\Phi_{n}^{*}$ angles determined from the relations

$$
\begin{gathered}
\cos \Phi_{n}^{*}=\frac{\tilde{Q}_{\theta n}}{\tilde{\rho}_{n}}, \quad \sin \Phi_{n}^{*}=\frac{\tilde{Q}_{\varepsilon n}}{\tilde{\rho}_{n}} \\
\tilde{Q}_{\theta n}=Q_{\theta n}+\frac{V_{b}}{V_{e}}\left(2 Q_{z n}^{2}-Q_{x n}^{2}-Q_{y n}^{2}\right) \\
+\frac{N_{e}}{V_{e}}\left(Q_{\varepsilon n}^{2}-Q_{\theta n}^{2}\right)+\frac{V_{e}^{R}}{V_{e}} Q_{\theta n}^{R}, \\
\tilde{Q}_{\varepsilon n}=Q_{\varepsilon n}+\frac{V_{b}}{V_{e}}\left(Q_{x n}^{2}-Q_{y n}^{2}\right) \\
+\frac{2 N_{e}}{V_{e}} Q_{\theta n} Q_{\varepsilon n}+\frac{V_{e}^{R}}{V_{e}} Q_{\varepsilon n}^{R},
\end{gathered}
$$

Table 3. Symmetrized distortions of nearest-neighbor oxygen environment of $\mathrm{Mn}^{3+}$ ions

\begin{tabular}{c|r|r|r|r|r|r|c}
\hline Ion & $Q_{\varepsilon}, \AA$ & $Q_{\theta}, \AA$ & $Q_{x}, \AA$ & $Q_{y}, \AA$ & $Q_{z}, \AA$ & $Q_{\theta}^{R}, \AA$ & $Q_{\varepsilon}^{R}, \AA$ \\
\hline Position 1 Mn1 & 0.27 & -0.14 & 0.13 & 0.14 & 0.39 & -0.107 & 0.12 \\
Position 1 Mn2 & 0.27 & -0.15 & 0.13 & 0.13 & 0.19 & -0.107 & 0.12 \\
Position 2 Mn3 & -0.37 & -0.16 & -0.05 & -0.63 & -0.39 & -0.04 & 0.25 \\
Position 2 Mn4 & -0.06 & 0.41 & 0.12 & 0.08 & 0.00 & -0.04 & 0.25 \\
\hline
\end{tabular}




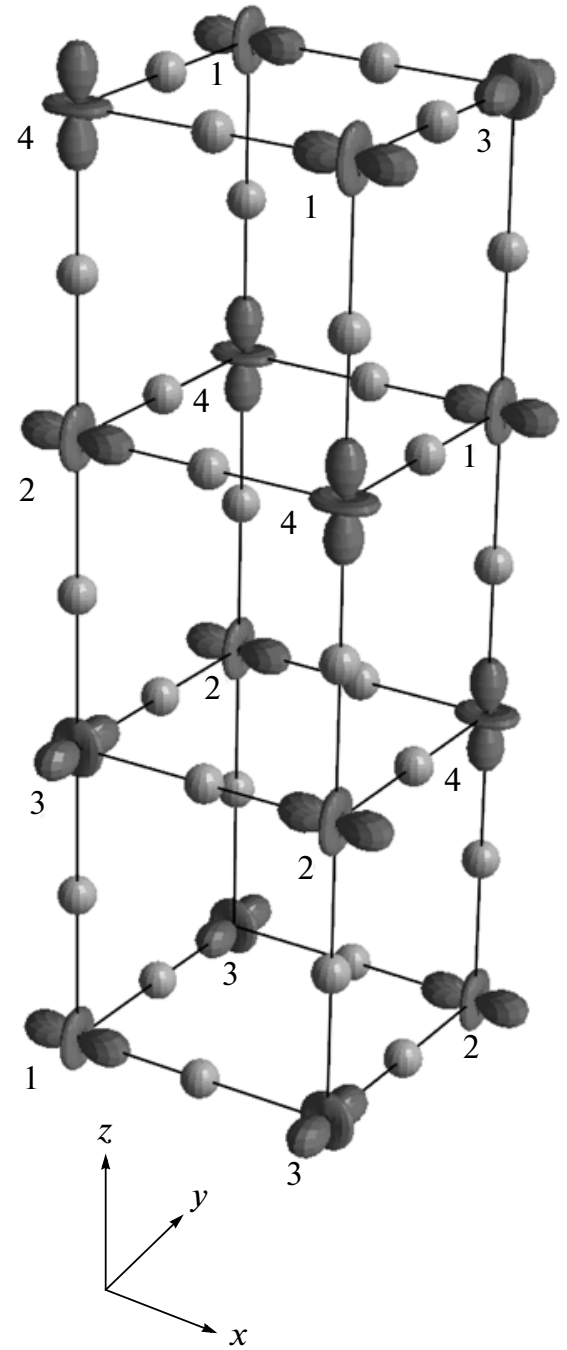

Fig. 1. Orbital structure of $\mathrm{BiMnO}_{3}$ representing manganese ions as electron densities and oxygen ions as spheres (for clarity, bismuth ions are omitted and octahedra axes are depicted as mutually orthogonal); numerals $1-4$ indicate manganese ions with different orbital states.

$$
\tilde{\rho}_{n}=\sqrt{\tilde{Q}_{\theta n}^{2}+\tilde{Q}_{\varepsilon n}^{2}}
$$

Figure 1 shows the results of modeling the orbital structure of $\mathrm{BiMnO}_{3}$ with allowance for linear contribution (3). The quantitative characteristics are presented in Table 4, from which it is seen that the maximum influence of the nonlinear contribution from $e_{g}$

Table 4. Mixing angles of orbital states of bismuth manganite

\begin{tabular}{c|c|c}
\hline $\begin{array}{c}\mathrm{Mn}^{3+} \text { ion } \\
\text { (see Fig. 1) }\end{array}$ & $\Phi_{n}$ & $\Phi_{n}^{*}$ \\
\hline 1 & $119.1^{\circ}$ & $\approx 120^{\circ}$ \\
2 & $119.7^{\circ}$ & $\approx 120^{\circ}$ \\
3 & $-112.9^{\circ}$ & $\approx-(105-116)^{\circ}$ \\
4 & $-7.9^{\circ}$ & $\approx-(3-18)^{\circ}$ \\
\hline
\end{tabular}

type distortions of the nearest-neighbor environment and the linear contribution from the next-to-nearest neighbor environment is manifested in the orbital state of $\mathrm{Mn}^{3+}$ ions in position 2. At the same time, the orbital state of ions in position 1 is subject to a small influence of the distortions of all types.

\section{MAGNETIC INTERACTIONS IN $\mathrm{BiMnO}_{3}$}

The type of magnetic structure (ferromagnetic vs. antiferromagnetic) of bismuth manganite can be modeled using the Hamiltonian of superexchange interaction:

$$
H_{\mathrm{ex}}=\sum_{l m} J_{l m}\left(\mathbf{S}_{l} \cdot \mathbf{S}_{m}\right) \text {. }
$$

Let us consider the superexchange interaction between nearest-neighbor $\mathrm{Mn}^{3+}$ ions, which depends on orbital states of interacting ions. In contrast to the case of rare-earth manganites [11], the orbital states of magnetic neighbors differ in both sign and magnitude. Then, the orbital dependence of the constants of superexchange interaction is as follows:

$$
\begin{gathered}
J_{z, l m}=\frac{J_{0} \cos ^{2} \varphi_{l m}}{r_{l m}^{10}}\left[1+\alpha\left(\cos \Phi_{l}^{*}+\cos \Phi_{m}^{*}\right)\right. \\
\left.+\beta \cos \Phi_{l}^{*} \cos \Phi_{m}^{*}\right] \\
J_{x(y), l m}=\frac{J_{0} \cos ^{2} \varphi_{l m}}{r_{l m}^{10}} \\
\times\left[1-\frac{\alpha}{2}\left(\cos \Phi_{l}^{*} \pm \sqrt{3} \sin \Phi_{l}^{*}\right.\right. \\
\left.+\cos \Phi_{m}^{*} \pm \sqrt{3} \sin \Phi_{m}^{*}\right) \\
+\frac{\beta}{4}\left(\cos \Phi_{l}^{*} \pm \sqrt{3} \sin \Phi_{l}^{*}\right) \\
\left.\times\left(\cos \Phi_{m}^{*} \pm \sqrt{3} \sin \Phi_{m}^{*}\right)\right]
\end{gathered}
$$

where $J_{0}=1.69 \times 10^{4} \mathrm{~K} \AA^{10}, \alpha=1.0, \beta=4.5$ [11], $\Phi_{i}$ is the angle of mixing of eigenfunctions of the orbital ground state of $\mathrm{Mn}^{3+}$ ion with wavefunction (1), $r_{l m}$ is the average distance in the interacting $\mathrm{Mn}-\mathrm{O}$ pair ( $l$ th and $m$ th ions), $\varphi_{l m}$ is the $\mathrm{Mn}-\mathrm{O}-\mathrm{Mn}$ bond angle (with values taken from experimental data [8]), and $x, y, z$ are the orientations of pseudoperovskite lattice axes (directed approximately along the bonds between nearest-neighbor manganese ions). Table 5 presents the values of exchange interaction constants calculated using the data from Table 4 . The mutual arrangement of ferromagnetic and antiferromagnetic links in the adopted model structure is shown in Fig. 2.

The pattern of mutual arrangement of ferromagnetic and antiferromagnetic links (with exchange interaction signs according to the Goodenough-Kan- 
amori rules), which could be determined using the known crystalline structure $[7,8]$, allowed the authors of earlier investigations $[1,7,8]$ to judge the existence of competition between exchange interactions and conclude that ferromagnetism is dominant. This conclusion was consistent with experimental data [7, 8] for the magnetic structure. Indeed, each manganese ion has four ferromagnetic and two antiferromagnetic links with the same order of interaction magnitudes. Note that antiferromagnetic links in this structure do not form continuous lines along any axis. Thus, ferromagnetic links are predominant. However, the presence of competition between superexchange interactions does not allow such an unambiguous conclusion. Optimization of the exchange contribution with allowance for values of the superexchange parameters (see Table 5) leads to an antiferromagnetic ( $A$ type) structure.

\section{RESULTS AND DISCUSSION}

Calculations of the magnetic structure of bismuth manganite performed in the vibronic interaction model including linear contribution (3) from only the nearest neighbors and superexchange with only the nearest neighbors, led to a magnetic structure of the $A$ type. This structure does not coincide with the ferromagnetic structure according to experimental data [7, 8], but it is possible to establish particular interactions that must be taken into account in order to adequately describe the experimental data.

Since the bismuth manganite crystal is strongly distorted, it is expedient to consider how the orbital structure modified by additional contributions (4) and (5) influences the exchange interaction. In other words, it is necessary to elucidate whether small changes in the orbital structure due to these contributions can shift the competition of exchange interactions toward the predominant ferromagnetism. In order to answer this, we have fitted the exchange interaction constants $\left(V_{b}, N_{e}, V_{e}^{R}\right)$ so that the orbital structure would correspond to a "boundary" state, in which the magnetic energy of the $A$-type structure would be equal to that of the $F$-type structure.

It has been found that this state is possible for the following values of the interaction constants:

$$
\begin{gathered}
V_{b}=-0.02 \mathrm{eV} / \AA^{2}, \quad V_{e}^{R}=-0.40 \mathrm{eV} / \AA^{2}, \\
N_{e}=-0.97 \mathrm{eV} / \AA^{2} .
\end{gathered}
$$

It is useful to compare these values to analogous data for $\mathrm{LaTiO}_{3}[15]$ :

$$
V_{b}=0.04 \mathrm{eV} / \AA^{2}, \quad V_{e}^{R}=-0.16 \mathrm{eV} / \AA^{2} .
$$

As can be seen, these values are of the same order of magnitude. The value of $N_{e}$ has no analog for comparison, but it is possible to estimate the value of $N_{e} \rho / V_{e} \approx$ 0.25 , which shows that this contribution is much smaller than the main linear term. A rather insignifi-
Table 5. Exchange interaction constants determined for bismuth manganite in approximation of linear vibronic interaction $\left(V_{b}, N_{e}, V_{e}^{R}=0\right)$ with nearest-neighbor environment (minus sign corresponds to ferromagnetic character of exchange)

\begin{tabular}{c|c|c|r|r}
\hline \multirow{2}{*}{$\begin{array}{c}\mathrm{Mn}^{3+} \text { ion } \\
(\text { see Fig. 1) }\end{array}$} & \multicolumn{4}{|c}{ Exchange with nearest neighbors } \\
\cline { 2 - 5 } & $\begin{array}{c}\text { interaction } \\
\text { with ion }\end{array}$ & $J_{x}, \mathrm{~K}$ & $J_{y}, \mathrm{~K}$ & $J_{z}, \mathrm{~K}$ \\
\hline \multirow{2}{*}{1} & 3 & -11 & -11 & 13 \\
& 4 & -13 & 13 & -6 \\
\hline \multirow{2}{*}{2} & 3 & -11 & -9 & 13 \\
& 4 & -13 & 13 & -7 \\
\hline
\end{tabular}

cant change in these constants makes a ferromagnetic structure predominating over the antiferromagnetic one. The values of interaction parameters for the "boundary" orbital structure are given in Table 6.

The distribution of electron densities did not change significantly as compared to that depicted in Fig. 1. The pattern also qualitatively corresponds to the Goodenough-Kanamori rules, but the quantitative balance of superexchange interactions shifted to ferromagnetic ordering. Thus, we may conclude on the significant effect of additional contributions (4) and (5), which is especially important in frustrated magnets.

As an alternative to the orbital mechanism, let us consider the possible influence of the exchange inter-

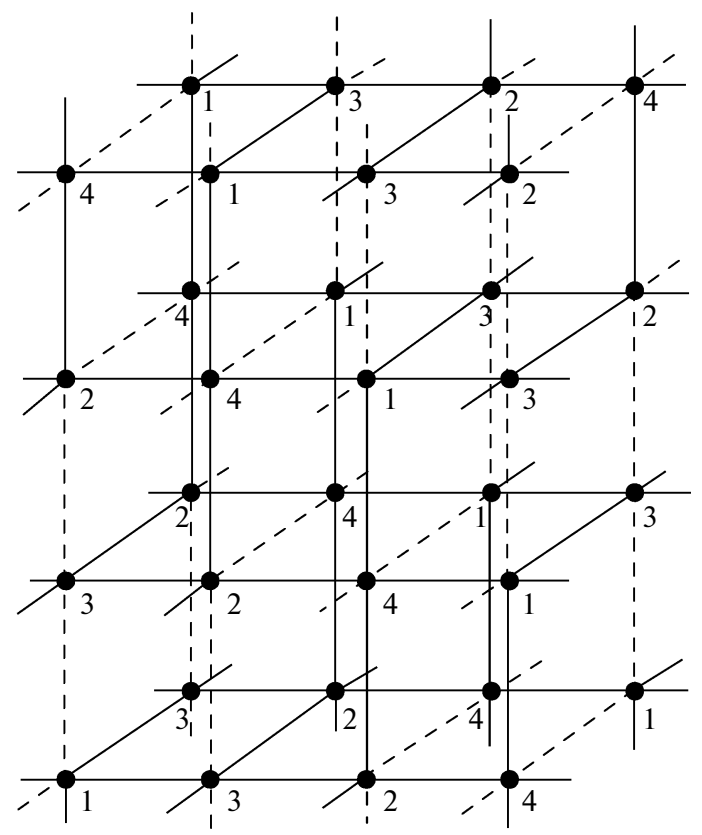

Fig. 2. Magnetic cell of $\mathrm{BiMnO}_{3}$. Ferromagnetic and antiferromagnetic links are depicted by solid and dashed lines, respectively; numerals 1-4 indicate manganese ions with different orbital states. 
Table 6. Mixing angles of orbital wavefunctions and superexchange interaction constants in bismuth manganite with refined orbital structure

\begin{tabular}{c|c|c|r|r|r}
\hline \multirow{2}{*}{$\begin{array}{c}\mathrm{Mn}^{3+} \text { ion } \\
\text { (see Fig. 1) }\end{array}$} & \multirow{2}{*}{$\Phi_{n}^{*}$} & \multicolumn{4}{|c}{ Exchange with nearest neighbors } \\
\cline { 3 - 6 } & $\begin{array}{c}\text { Interaction } \\
\text { with ion }\end{array}$ & $J_{x}, \mathrm{~K}$ & $J_{y}, \mathrm{~K}$ & $J_{z}, \mathrm{~K}$ \\
\hline \multirow{2}{*}{1} & $120^{\circ}$ & 3 & -17 & -10 & 11 \\
& & 4 & -17 & 11 & -10 \\
\hline \multirow{2}{*}{2} & $122^{\circ}$ & 3 & -17 & -8 & 11 \\
& & 4 & -16 & 11 & -12 \\
\hline \multirow{2}{*}{3} & $-109^{\circ}$ & 1 & -17 & -10 & 11 \\
& & 2 & -17 & -8 & 11 \\
\hline \multirow{2}{*}{4} & $-11^{\circ}$ & 1 & -16 & 11 & -12 \\
& & 2 & -17 & 11 & -10 \\
\hline
\end{tabular}

action between next-to-nearest magnetic neighbors on the stabilization of ferromagnetic ordering. For this effect to take place, it suffices to have a small value of $J_{n n n} \approx-2 \mathrm{~K}$. However, the change in the superexchange interaction constants due to refinement of the orbital structure turns out to be rather large (from 1 to $6 \mathrm{~K})$. The exchange interaction between next-to-nearest magnetic neighbors has also been studied in other manganites [16], but it proved to have an antiferromagnetic character. Therefore, this contribution must be excluded from consideration.

In concluding, it should be noted that our investigation of the effect of orbital ordering on the magnetic structure of bismuth manganite led to the conclusion that nonlinear contributions and the contributions due to next-to-nearest neighbors in the Hamiltonian of vibronic interaction play a significant role in the formation of ferromagnetic ordering in this crystal. This influence is related to the presence of competition between superexchange interactions. In lanthanum manganite [11], where the antiferromagnetic ( $A$-type) structure is stable and unambiguous, a change in the mixing angles of orbital functions by several degrees can lead to a similar modification of the exchange parameters, but the type of magnetic ordering will remain unchanged.

\section{REFERENCES}

1. A. Moreira dos Santos, S. Parashar, A. R. Raju, Y. S. Zhao, A. K. Cheetham, and C. N. R. Rao, Solid
State Commun. 122 (1-2), 49, (2002); A. Moreira dos Santos, A. K. Cheetham, T. Atou, Y. Syono, Y. Yamaguchi, K. Ohoyama, H. Chiba, and C. N. R. Rao, Phys. Rev. B: Condens. Matter 66 (6), 064425 (2002).

2. C. G. Zhong, J.-H. Fang, and Q. Jiang, J. Phys.: Condens. Matter 16, 9059 (2004).

3. D. I. Khomskii, J. Magn. Magn. Mater. 306, 1 (2006).

4. M. Grizalez, E. Martinez, J. Caicedo, J. Heiras, and P. Prieto, Microelectron. J. 39 (11), 1308 (2008).

5. I. V. Solovyev and Z. V. Pchelkina, New J. Phys. 10, 073021 (2008).

6. I. V. Solovyev and Z. V. Pchelkina, Phys. Rev. B: Condens. Matter 82, 094425 (2010).

7. A. A. Belik, S. Iikubo, T. Yokosawa, K. Kodama, N. Igawa, Sh. Shamoto, M. Azuma, M. Takano, K. Kimoto, Y. Matsui, and E. Takayama-Muromachi, J. Am. Chem. Soc. 129 (4), 971 (2007).

8. D. P. Kozlenko, A. A. Belik, S. E. Kichanov, I. Mirebeau, D. V. Sheptyakov, Th. Strässle, O. L. Makarova, A. V. Belushkin, B. N. Savenko, and E. TakayamaMuromachi, Phys. Rev. B: Condens. Matter 82 (1), 014401 (2010).

9. A. P. Pyatakov and A. K. Zvezdin, Phys._Usp. 55 (6), 557 (2012).

10. A. A. Belik, K. Kodama, N. Igawa, Shin-ichi Shamoto, K. Kosuda, and E. Takayama-Muromachi, J. Am. Chem. Soc. 132 (23), 8137 (2010); A. Castro, C. Correas, O. Peña, A. R. Landa-Cánovas, M. Algueró, H. Amorín, M. Dollé, El. Vila, and T. Hungría, J. Mater. Chem. 22 (19), 9928 (2012).

11. L. E. Gontchar and A. E. Nikiforov, Phys. Rev. B: Condens. Matter 66, 014437 (2002).

12. A. E. Nikiforov, S. Yu. Shashkin, M. L. Levitan, and T. H. Agamalyan, Phys. Status Solidi B 118, 419 (1988).

13. A. A. Mozhegorov, A. V. Larin, A. E. Nikiforov et al., Phys. Rev. B: Condens. Matter 79, 054418 (2009).

14. A. E. Nikiforov, S. E. Popov, and S. Yu. Shashkin, Phys. Met. Metallogr. 87 (2), 97 (1999).

15. A. A. Mozhegorov, A. E. Nikiforov, A. V. Larin, A. V. Efremov, L. E. Gonchar', and P. A. Agzamova, Phys. Solid State 50 (9), 1724 (2008).

16. T. Kimura, S. Ishihara, H. Shintani, T. Arima, K. T. Takahashi, K. Ishizaka, and Y. Tokura, Phys. Rev. B: Condens. Matter 68 (6), 060403(R) (2003).

Translated by P. Pozdeev 